\title{
Prominence Oscillations and the Influence of the Distant Photosphere
}

\author{
N.A.J. Schutgens, M. Kuperus and G.H.J. van den Oord \\ Astronomical Institute Utrecht, Utrecht University, the Netherlands
}

\begin{abstract}
We model vertical prominence dynamics, describing the evolution of the magnetic field in a self-consistent way. Since the photosphere imposes a boundary condition on the field (flux conservation), the Alfvén crossing time $\tau_{0} / 2$ between prominence and photosphere has to be taken into account. Using an electrodynamical description of the prominence we are able to compare two basic prominence models: Normal Polarity (NP) and Inverse Polarity (IP).

The results indicate that for IP prominences, the stability properties are sensitive to $\omega \tau_{0}$ ( $\omega$ : oscillation frequency of prominence). For $\omega \tau_{0} \gtrsim 1$ instability results. Forced oscillations of five minutes are efficiently excited in IP prominences that meet certain criteria only. NP prominences on the other hand, are insensitive to the Alfvén crossing time. Forced oscillations of five minutes are difficult to excite in NP prominences.
\end{abstract}

\section{Introduction}

For typical quiescent prominence heights $\left(z_{0} \approx 30,000 \mathrm{~km}\right)$ and coronal Alfvén speeds $\left(v_{A} \approx 100-10,000 \mathrm{~km} / \mathrm{s}\right)$, the Alfvén crossing time is $\tau_{0} / 2=z_{0} / v_{A} \approx$ $0.1-10 \mathrm{~min}$., which is of the same order as observed short oscillation periods in prominences (Yi Zhang et al. 1991, Thompson and Schmieder 1991). If the photosphere is instrumental in the support of a prominence, the Alfvén crossing time has to be taken into account when studying such oscillations (van den Oord and Kuperus 1992). Basically, the time-scale $\tau_{0} / 2$ is the shortest timescale on which photosphere and prominence can 'communicate'. It represents the delay with which the photospheric boundary condition influences the prominence dynamics.

Kuperus and Raadu (1974) suggested that the supporting force on a prominence stems from flux conservation at the photospheric level. The field due to the prominence current cannot enter the photosphere and the filament is effectively repelled. For a bipolar region, the Kuperus-Raadu model is the typical example of an Inverse Polarity (IP) prominence. Kippenhahn and Schlüter (1957) proposed a more local support mechanism: a small dip in the field lines of a coronal arcade would be sufficient to trap matter and support it against gravity. The resulting field topology is known as Normal Polarity (NP). Flux conservation at the photospheric level plays only a minor role in the support of NP prominences.

An equation of motion for an IP prominence, that takes the delayed photospheric boundary condition into account, has been derived and solved in the 
linear approximation (Schutgens 1997a). We present here a summary of a comparative study into the vertical dynamics of IP and NP prominences (Schutgens 1997b).

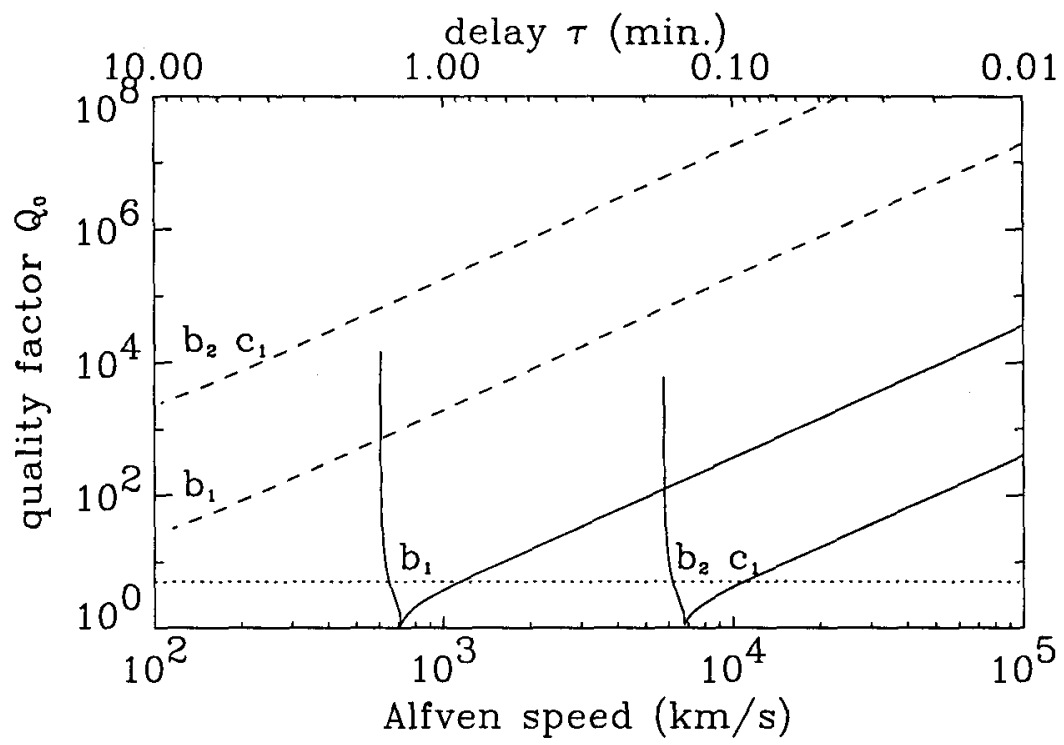

Figure 1. The quality factors of stable (!) IP (solid lines) and NP (dashed lines) prominence oscillations as a function of the Alfvén speed. The assumed values for the prominence longitudinal density $m$ and the arcade field strength $B_{0}$ are $\left.b_{1}\right) m=3.1 \times 10^{4} \mathrm{~kg} / \mathrm{m}, B_{0}=0.001 \mathrm{~T} ; b_{2}$ ) $\left.m=3.1 \times 10^{2} \mathrm{~kg} / \mathrm{m}, B_{0}=0.001 \mathrm{~T} ; c_{1}\right) m=3.1 \times 10^{4} \mathrm{~kg} / \mathrm{m}, B_{0}=0.01 \mathrm{~T}$.

\section{Free Oscillations}

Consider a prominence in equilibrium, which experiences an impulsive perturbation and starts to oscillate. Our model allows us to study frequency and stability properties of IP and NP prominences as a function of the Alfvén speed. It turns out that the oscillation frequency $\omega$ depends only weakly on the Alfvén crossing time, and is fairly accurately approximated by the frequency found in the quasi-stationary approximation $\left(\tau_{0}=0\right): \omega \approx \omega_{\text {stat }}$. Also, the frequencies of NP prominences are usually one or more orders of magnitude smaller than those of IP prominences of the same longitudinal mass density.

The effect of the crossing time $\tau_{0} / 2$ on IP and NP stability properties can be analysed using the quality factor $Q_{0}=\omega / 2 \nu$, where $\omega$ is the frequency and $\nu$ the damping rate of the oscillation. A small $Q$ implies a strongly damped oscillation. Note that we do not apply any mass friction or wave emission; the damping 
results solely from the inclusion of a delayed photospheric boundary condition. Figure 1 shows that the oscillations in IP prominences become strongly damped $(Q \leqslant 1)$ for specific values of $v_{A}$. Also, left of the vertical asymptote present in each IP graph, the IP prominence is unstable. This instability occurs for $\omega \tau_{0} \approx 1$. NP prominences are only weakly damped and almost marginally stable $(Q \gtrsim 100)$.
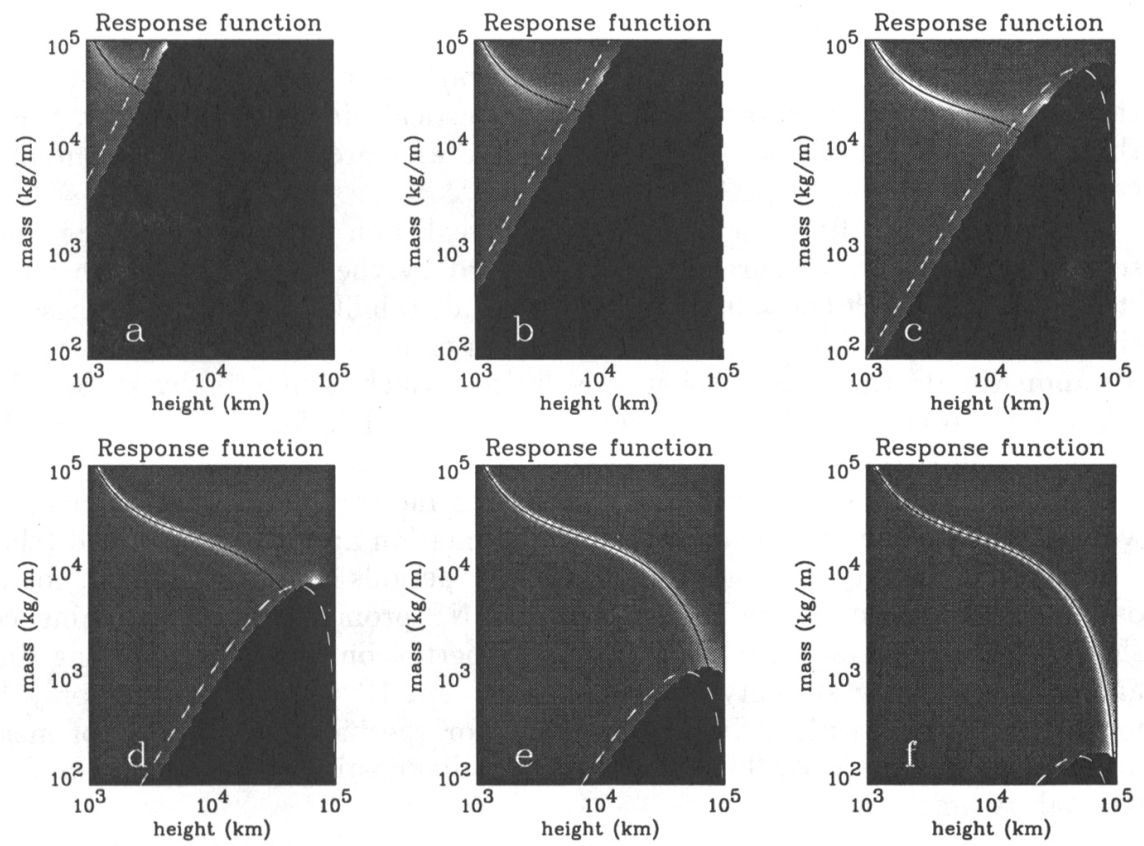

Figure 2. Gray-scale plot of the response function of forced oscillations in IP prominences for a driving period of $5 \mathrm{~min}$, for different Alfvén speeds; a) $\left.\left.v_{A}=100 \mathrm{~km} / \mathrm{s} ; b\right) v_{A}=251 \mathrm{~km} / \mathrm{s} ; c\right) v_{A}=631 \mathrm{~km} / \mathrm{s}$; d) $\left.\left.v_{A}=1585 \mathrm{~km} / \mathrm{s} ; e\right) v_{A}=3981 \mathrm{~km} / \mathrm{s} ; f\right) v_{A}=10,000 \mathrm{~km} / \mathrm{s}$. The black area shows prominences that are unstable. The response function is plotted as a gray-scale, with white denoting the largest response.

\section{Forced Oscillations}

Typical oscillation periods for IP and NP prominences are 1-10 min. and 10-100 minutes, respectively. As a consequence, harmonic forcing with a period of five min. can possibly excite IP prominences, but not NP prominences. The response of an IP prominence to such forcing (the amplitude of oscillation) is plotted in Figure 2, for different values of the Alfvén speed $v_{A}$. Here the black area represents an unstable, growing oscillation, while the white area denotes stable prominences that are very strongly excited by five min. forcing. The elongated white curve extending from the upper left corner, corresponds to filaments whose 
quasi-stationary oscillation period is equal to five min. Actually, the strongest response is obtained for those prominences that are associated with the bright little dot in Figure 2a, b, $c$ and d, just at the border of stable and unstable oscillations. Apparently prominences with a specific combination of height $z_{0}$, longitudinal density $m$ and coronal Alfvén speed $v_{A}$ show a very strong response.

\section{Conclusions}

We show that the Alfven crossing time-scale $\tau_{0} / 2$ is an important time-scale for short period prominence oscillations in the vertical direction. Basically, this is the shortest time-scale on which photosphere and prominence can 'communicate'.

The oscillation frequencies depend only weakly on the Alfvén crossing timescale, they are fairly accurately approximated by the frequency in the quasistationary approach $\left(\tau_{0}=0\right.$ or $\left.v_{A} \rightarrow \infty\right)$. The stability properties of these oscillations, however, are sensitive to the Alfvén crossing time-scale. For $\omega \tau_{0} \ll 1$ we approximate the quasi-stationary solution, which is marginally stable. For $\omega \tau_{0} \approx 1$ the oscillations become strongly damped, while for $\omega \tau_{0} \gtrsim 1$ the oscillations grow in time.

For typical parameter values of prominence and corona, NP prominences are well described in the quasi-stationary approximation and weakly damped (close to marginal stability, $\omega \tau_{0} \ll 1$ ). Since their periods are much longer, forced oscillations of five min. are hard to excite in NP prominences. IP prominences show a strong dependence of the stability properties on the Alfvén crossing time and can even loose stability $\left(\omega \tau_{0}=0.1-10\right)$. An IP prominence can only be forced to oscillate with a five min. period for specific combinations of mass, height and Alfvén speed. This can serve as a diagnostic for prominence and/or coronal parameters.

The case of horizontal oscillations is currently under investigation.

\section{References}

Kippenhahn, R. and Schlüter, A. 1957, ZAp 43, 36

Kuperus, M. and Raadu, M.A. 1974, Sol. Phys., 31, 189

Schutgens, N.A.J. 1997a, A\&A, 323, 969

Schutgens, N.A.J. 1997b, A\&A, 325, 352

van den Oord, G.H.J. and Kuperus, M. 1992, Sol. Phys., 142, 113

Thompson W.T. and Schmieder B. 1991, A\&A 243, 501

Yi Zhang, Engvold O. and Keil S.L. 1991, Sol. Phys., 132, 63 